\title{
Study of crushing of grains of limestone under the effect of cyclic loading with the fractal dimension
}

\author{
Bouzeboudja $\mathrm{A}^{1,{ }^{*}}$, Melbouci $\mathrm{B}^{1}$, Bouzeboudja $\mathrm{O}^{2}$
}

1 Geomaterials Laboratory, Environment and Development, University of Tizi-Ouzou, 15000, Algeria.
2 Laboratory of analysis and modeling of random phenomena, University of Tizi-Ouzou, 15000, Algeria.
* Corresponding Author: bouzeboudjaa5@gmail.com

Received: 15-02-2018

Revised: 16-05-2018

Accepted: 20-05-2018

\begin{abstract}
The grains can be of very diverse nature (size, shape, mineralogy, etc), indeed even if each grain is a fairly simple object, the set of grains is a very complex system in which the geometrical and physical properties of the grains, their forces and their orientations play a crucial role in their mechanical behavior. The measurement of their shape in soil mechanics has historically required the use of standards and diagrams to which the different grains can be compared. However, these methods are approaches and remain insufficient and incomplete for the actual measurement of their form and the understanding of their behavior remains a task far from being completed.

This work is in this context and proposes to analyze the shape and size of the grains with the fractal theory using the Box Counting method. It also makes it possible to study their influence on the crushing of the grains of the calcareous material under the effect of the cyclic loading-unloading during the oedometer test. The obtained results show that the variation of the fractal dimension, corresponding to the crushing effect of the grains, is strongly influenced by their shape, their size, their particle size distribution and the variation in the number of loading-unloading cycles.
\end{abstract}

Key words: Cyclic loading, geometrical characteristics of grains, crushing, fractal dimension.

\section{Introduction}

The granular materials may undergo a rupture or crushing phenomenon under the effects of shear, compression or compaction and even under the effect of a small projectile. This phenomenon causes their fragmentation in different aspects.

When the grains are solid, hard and fairly rounded, they can withstand high stresses which require, for example, the use of heavy equipment or compactors in the construction of high dams in earth and riprap to meet the requirements of high density. On the other hand, the angularly shaped grains of freshly extracted quarry materials undergo fragmentation due to the breakage of the asperities under lesser stresses and reach higher densities (Ramamurthy, 1969).

Soil, which is both a fragmentary and porous medium, has a fairly complex structure where a same fractal representation may be particularly suitable. It is then necessary to characterize the shape of the grains using the fractal dimension (Chen et al., 1996).

\section{Fractal dimension}

\subsection{Definition}

To measure a length, a surface or a volume, a common method consists in covering these sets of blocks whose length, surface or volume are taken as units of measure. This is precisely what is done with the classical methods when calculating these quantities by means of the euclidean dimension. The fractal dimension is the complement which was lacking in the latter as well as in the crystal symmetry in order to obtain results very close to reality (Falconer, 1990; Mandelbrot, 
1983). Indeed, fractal geometry can be used for the characterization of the soil. To calculate the fractal dimension, image analysis techniques are important after photographing the grains (Sezer et al., 2008).

\subsection{Box Counting (BC) method}

This method was defined in the work of Russel in 1980 (Russel et al., 1980), is the most frequently used and most popular in practice. In the case of "fragmentation", the fractal dimension calculated by this method gives us an idea of the dimensional distribution of the grains in the soil and the fragmentation process. Mandelbrot showed that the distribution of rock fragments is a fractal distribution. As he also suggested that fractal fragmentation could be measured by developing the fractal dimension of equation (1).

This method consists in dividing the image of a grain into small squares of identical dimensions (making a mesh), thus the outline of the grain passing through these boxes is counted, and we do the same operation but this time with boxes of decreasing sizes and so on...

This method is based on the premise that the image of the grain is the number of boxes based on their sizes, and this relationship is represented by the following formula:

$\mathrm{x}$ : size of the boxes;

$$
\mathrm{N}(\mathrm{X}>\mathrm{x})=\mathrm{Kx}^{-\mathrm{FD}}
$$

$\mathrm{X}$ : linear dimension of the grains greater than the dimension $\mathrm{x}$;

$\mathrm{N}(\mathrm{X}>\mathrm{x})$ : number of boxes;

$\mathrm{k}$ : constant of proportionality;

$\mathrm{FD}_{\mathrm{R}}$ : fractal dimension of fragmentation (Huang \& Zhan, 2002; Wang et al., 2006).

By plotting these values; size boxes depending the number of boxes in a logarithmic graph, the fractal dimension is obtained according to the slope best suited to the regression line and can be calculated by the following equation:

$$
\mathrm{FD}_{\mathrm{R}}=-\mathrm{m}
$$

$\mathrm{m}$ : The exponent of the line best suited to the scatter of points (Figure 1).

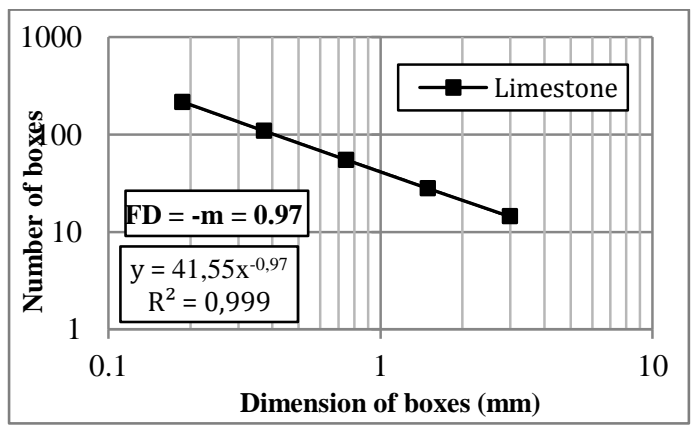

Fig 1. Calculating the fractal dimension of an angular limestone grain, size $6.3 \mathrm{~mm}$, using the Box Counting method.

\section{Material studied}

The material used in this study is a local material, most encountered during the various civil engineering works (buildings, roads, bridges, dams, watertight barriers, etc.), which is limestone (Figure 2) a mineral substance characterized by a chemical composition in which calcium carbonate (CaCO3) predominates, often of organic origin (limestones with foraminifers including chalk, shellstone limestones), but also of chemical origin (calcite, eolithic limestone, pisolithic, lithographic). 
The limestone used is of Jurassic age, extracted from a deposit located at a distance of $6 \mathrm{~km}$ from the city of Bejaia (12 km west of the city on the coast).

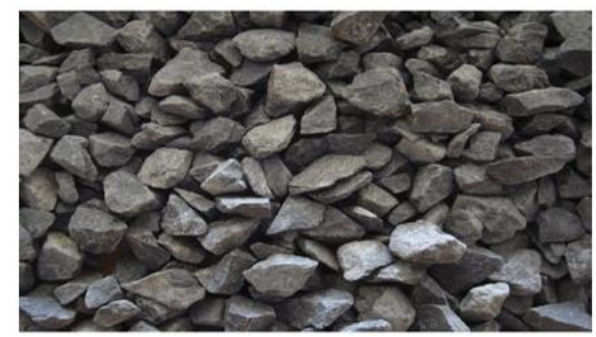

Fig 2. Limestone studied.

The chemico-mineralogical compositions of the limestone are grouped in Table 1.

Table 1. The chemico-mineralogical compositions of the material used.

\begin{tabular}{|l|l|c|c|}
\hline Compounds & Quartz \% & Calcium oxide \% & PAF \% \\
\hline \hline Limestone & 1 à 5 & 55 à 63 & 35 à 42 \\
\hline
\end{tabular}

The physico-mechanical properties of this rock are represented in Tables 2 and 3.

Table 2. The physical characteristics of the material used.

\begin{tabular}{|l|l|l|l|l|}
\hline \multirow{2}{*}{ Characteristics } & $\begin{array}{l}\text { Optimal volumetric } \\
\text { weight } \gamma_{\text {opt }}[\mathrm{kN} / \mathrm{m} 3]\end{array}$ & $\begin{array}{l}\text { Optimal water } \\
\text { content } \mathrm{W}_{\text {opt }}[\%]\end{array}$ & $\begin{array}{l}\text { Porosity n } \\
{[\%]}\end{array}$ & $\begin{array}{l}\text { Volumetric weight of } \\
\text { solid grains } \gamma_{\mathrm{s}}[\mathrm{kN} / \mathrm{m} 3]\end{array}$ \\
\hline \hline Limestone & 21,6 & 5,1 & 9 & 27,4 \\
\hline
\end{tabular}

Table 3. The mechanical characteristics of the material studied.

\begin{tabular}{|l|l|l|l|l|}
\hline Characteristics & Fragmentability FR & Degradability DG & Micro-Deval MDE & Los-Angeles LA [\%] \\
\hline Limestone & 2 & 1.03 & 12.5 & 20 \\
\hline
\end{tabular}

The samples were made in such a way that each test specimen is made up of a single form of grain. Three forms of grains were visually selected (Figure 3): the form-rounded (roughly rounded, with little angles and are very open), the angular shape (with several sharp edges) and the elongated shape (where the length of the grain is at least 1.5 times its width).

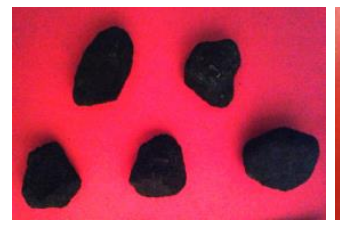

(a)

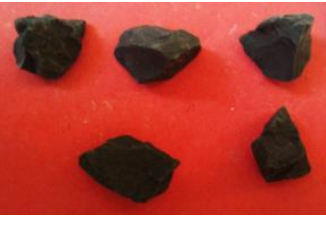

(b)

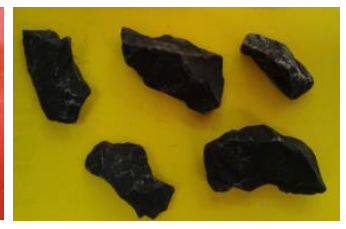

(c)

Fig 3. Forms used: (a) sub-rounded, (b) angular and (c) elongated.

Three particle size classes were chosen for this study: 3.15/5, 3.15/8 and 5/8 mm (Figure 4).

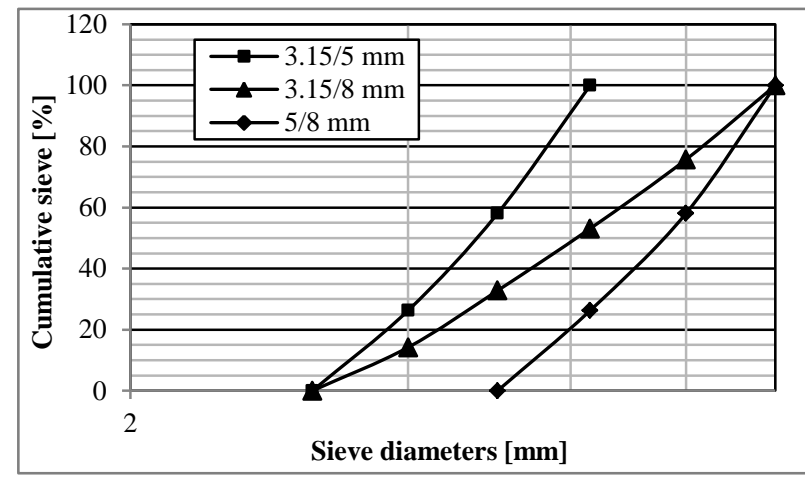

Fig 4. Granulometric curves before testing of the different classes for the material studied. 
Five control grains of each diameter of the three classes were stained to better evaluate their fragmentation after crushing at odometric tests, which are shown in the Figure 5. A good resolution camera was used to demonstrate better analysis of grain images before and after each test. The images are processed using MATLAB 7.9.0 (R2009b) software and the results of the fractal dimension obtained are interpreted using Excel.

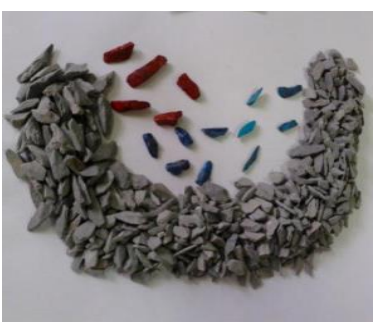

(a)



(b)

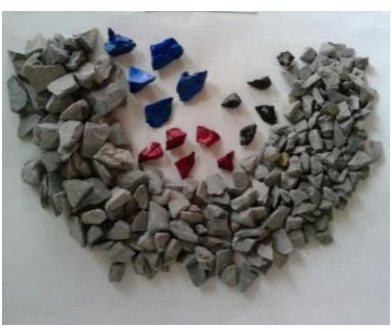

(c)

Fig 5. Different granulometric classes studied: (a) 3.15/5, (b) 3.15/8 and (c) 5/8 mm of the limestone used.

\section{Tests conducted}

The samples are subjected to oedometric tests (Figure 6), applying loading cycles of up to 6400 $\mathrm{kPa}$ followed by unloading cycles up to $700 \mathrm{kPa}$ (AFNOR, 1997). The tests are carried out using an automated Geocomp Corporation machine $(\varnothing=63.5 \mathrm{~mm})$. Vertical deformations or settlements of the sample in the tests are measured using a comparator and recorded using software.

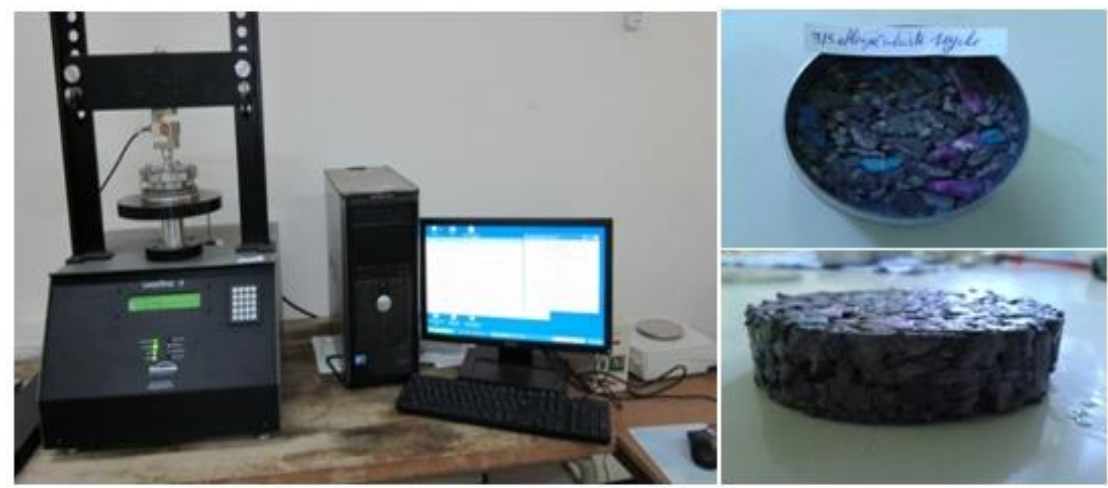

Fig 6. Controlled odometric device, Geocomp Corporation type.

\section{Presentation and interpretation of test results}

\subsection{Results and analysis}

This study is carried out with the aim of understanding the behavior and determining the mechanical characteristics and the influence of some parameters (namely the nature, shape and size of the grains and the applied load) by making oedometer tests on samples reconstituted in the laboratory using natural local material "limestone"; while taking account of the crush phenomenon in this trial.

The results are presented as oedometer curves. The behavior of granular materials is strongly influenced by the degree of crushing and grain breakage. Thus, the most important factor affecting the resistance to compressibility stresses is the phenomenon of fragmentation undergone by the granular body.

Actually, during our tests, the samples underwent deformations and ruptures of their grains. The amount of fines produced increases with increase of the load intensity. 
We recall that the placement of the samples is identical for all the tests and that each test was repeated several times in such a way that confirm systematically the reproducibility of the results obtained and to quantify the mechanical characteristics of the granular materials.

In our case, this test is used mainly to study the crushing of the grains of local material. The load is applied in stages of loading-unloading, with the presence of water. The deformations are almost instantaneous and the index of the voids decreases with the increase of the stress. If we represent the variation of the index of voids as a function of the effective stress $\sigma^{\prime}$ applied on the surface of the sample, we obtain the curves called odometric curves of compressibility (Figure 7).

During the tests, the material are crushed increasingly with the increasing number of cycles ranging from 1 to 5 cycles (Figure 7), regardless of the granular class and the shape of the grains. This creates a better compactness with optimum rearrangement of the grains and therefore a gradual decrease of the void ratio. Indeed, with the increase in the content of fines, the volume of the voids decreases. The compressibility curves of all the samples studied have practically a lowslope portion at the start of loading, followed by a steep portion beyond the pre-consolidation constraint.

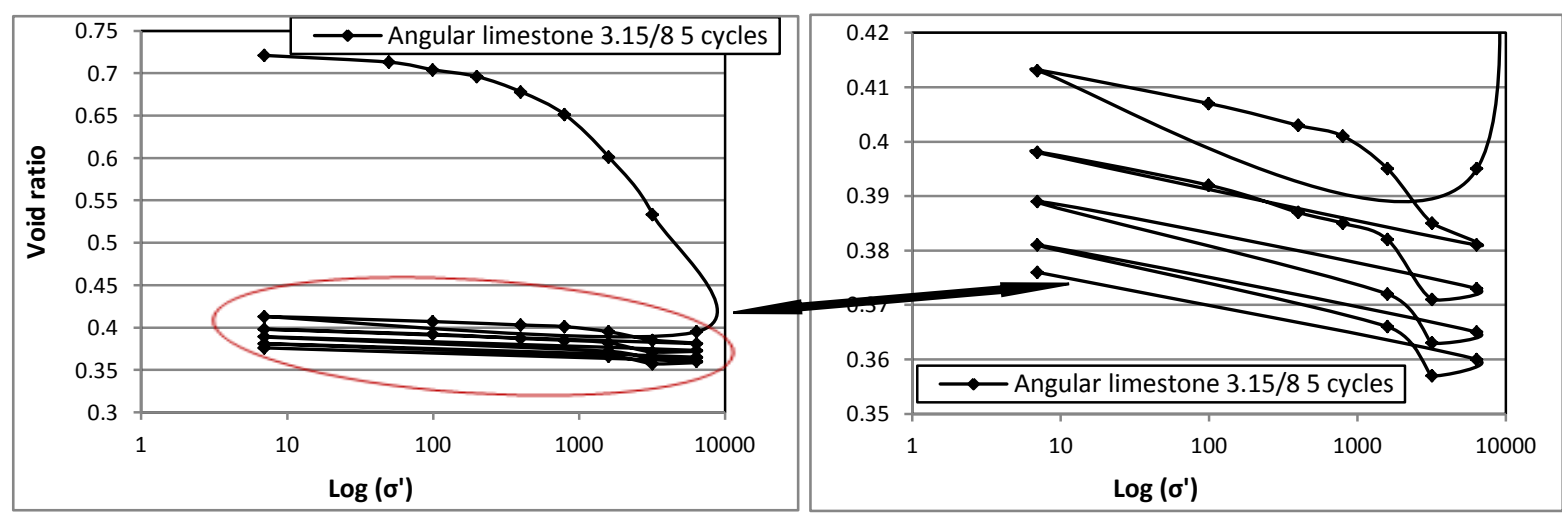

Fig 7. Odometric curves of the angular class $3.15 / 8 \mathrm{~mm}$, after 5 cycles of loading-unloading at $6400 \mathrm{kPa}$.

When a granular mass is subjected to a compression load, the grains resist the load through a series of contacts there between. Some grains may have more contacts than others, so they carry a greater load, which explains why they crash more than others. Fragmentation begins when highly stressed grains can no longer resist, breaking after that into small pieces. Some of these pieces move in the voids of the original structure of the material, which generates other chains of force and other assemblies of grains, by keeping high loads of microstructures appearing in the vicinity of the contacts of the grains, which can lead to a modification of the granular structure.

Note that the settlement under static loading is more important than the initial compactness is loose. Thus, settlements occur very quickly in a coarse medium, so a sufficiently permeable medium.

\subsection{Particle size analysis}

Grain crushing affects the characteristics of granular materials particularly on the spreading of granulometric curves.

In order to quantify the breakage of the grains and to demonstrate this crushing phenomenon in the tests carried out, several quantitative measurements of the rupture rate have been proposed (Lade et al., 1996 \& Einav, 2007a). The idea is to develop a quantitative parameter used to compare between various break levels, relative to a reference size. This reference may be the initial particle size, a supposed ultimate particle size or simply a given limit size. Indeed, a 
particle size analysis before and after each test was carried out and compared with the initial granulometric curve (Figure 8).

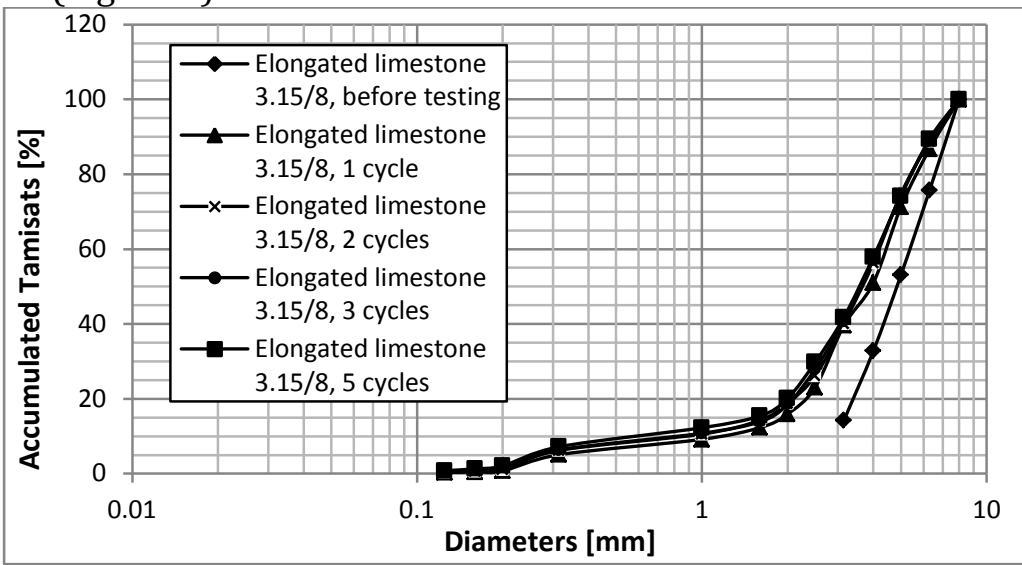

Fig 8. Particle size distribution curves of the elongated class $3.15 / 8 \mathrm{~mm}$, after 5 cycles of loading-unloading at $6400 \mathrm{kPa}$.

In addition, we observed that the higher is the number of loading/unloading cycles (Figure 8), the more significant is the crushing. Indeed, depending on the increase in the intensity of the stress. This crushing results in a reduction in the size of the grain with a modification of its shape and its surface state. What has been noticed even for limestone samples considered as a durable and hard material (Figure 9).
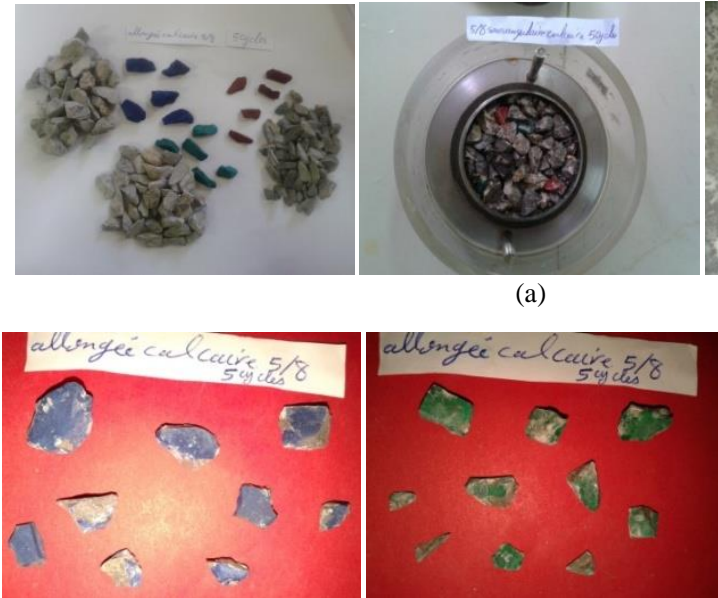

(c)

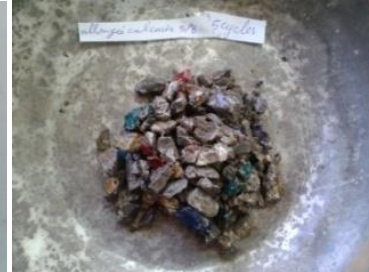

(b)

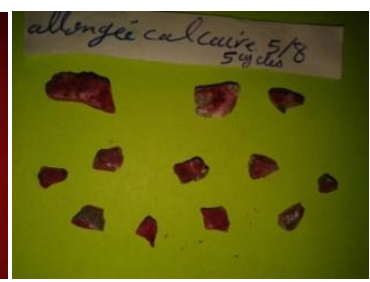

05) loading-unloading

Fig 9. Granulated crushing of the $5 / 8 \mathrm{~mm}$ classe of the elongated limestone after five (05) loadin
cycles at the oedometer test; (a) before the testing; (b) and (c) after the testing.

It is interesting to note that the fragmentation took place at the contact points of the grains subjected to the various stresses. When crushing occurs, a production of fines ensues. Thinner fragments begin to move the larger grains, as they can slip or roll without too much difficulty around other grains.

\subsection{Calculation of percentage of fines}

The Figure 10 shows that the quantity of fines produced increases with the number of loadingunloading cycles applied to the oedometer test, regardless of the shape of the grains and the granular class.

Indeed, the number of crushed grains is a function of the number of cycles. This quantity is larger for the sub-rounded shape; also it is close to the level at first and second cycles of loadingunloading for the sub-rounded and angular shapes, the percentage of fines produced by the 
grains of the elongated form is smaller compared to that obtained from the two other forms, for class 3.15/8 mm (Figure 10.a).

The amount of fragments produced by the angularly shaped limestone grains is smaller compared to fines obtained from of the other two forms, the fines content is higher for the elongated form than for the other two forms, for classes $5 / 8 \mathrm{~mm}$ and $3.15 / 5 \mathrm{~mm}$ after the oedometric test (Figures 10.b and 10.c).

The angular structure of the limestone grains produces many fines at each stage of the crushing.

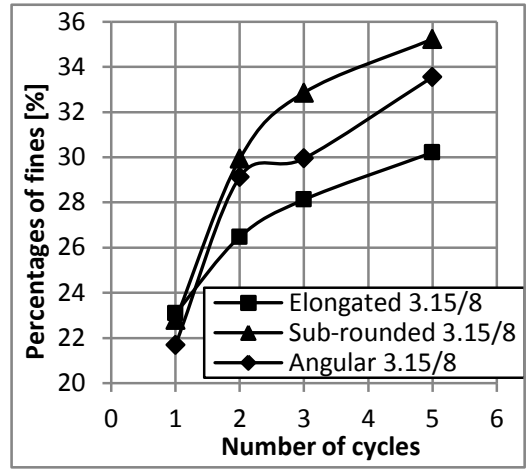

(a)

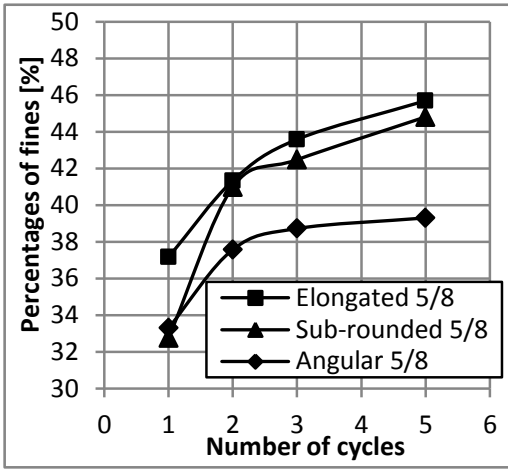

(b)

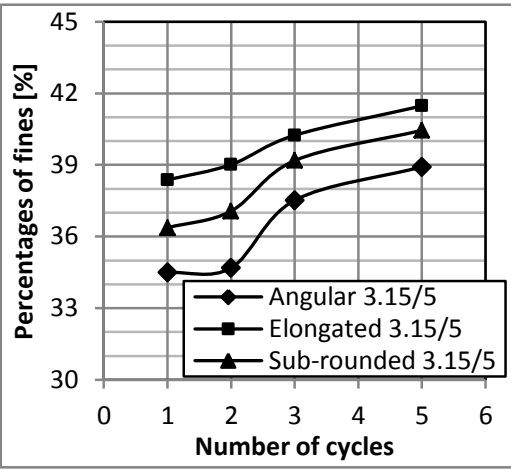

(c)

Fig 10. Percentage change of the fines based on the number of cycles for the three forms of limestone of classes $3.15 / 8,5 / 8$ and $3.15 / 5 \mathrm{~mm}$ under the oedometer test.

\section{Fractal dimension calculated before and after testing}

The introduction of fractal theory has improved the understanding of grain crushing and encouraged the application of fractal dimension in civil engineering work. The characteristics of the resistance at the oedometric test, as well as the different crushing rates observed during the tests, made it possible to understand the variations of the properties and the limitations of the studied granular material (Bouzeboudja \& Melbouci, 2016a; Bouzeboudja et al., 2016b; Ovalle, 2013).

For this method, the calculated fractal dimension is called the fractal dimension of roughness $\left(F D_{R}\right)$; it depends on the following parameters:

- The shape (sub-rounded, elongated and angular) and the size (large or small) of the grain studied;

- The scale of measurement, the larger the scale, the more precise the fractal dimension;

- The chosen face of the grain for imaging;

- The quality of the image taken (number of pixels).

To calculate the fractal dimension of roughness of the grains of the different samples studied with the Box Counting method before and after crushing; a good resolution camera was used in order to have a better analysis of the contours of the five colored grains of each diameter; and the images were processed using Auto-CAD software.
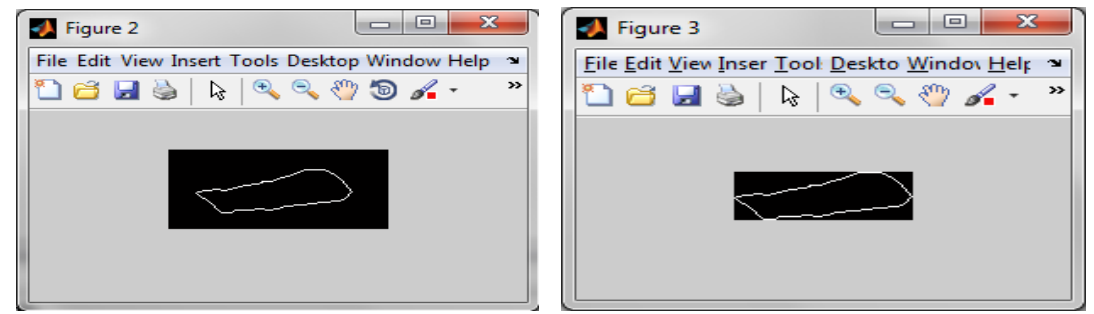

Fig 11. Calculating the fractal dimension of roughness with MATLAB R2009b by the Box Counting method. 
To facilitate $\mathrm{FD}_{\mathrm{R}}$ calculation, MATLAB R2009b software was used. By decreasing more and more the number of pixels of the calculation box, one gets closer to the actual contour of the grain. An example of the calculation is shown below (Figure 11), which is of interest for an elongated limestone of $\emptyset=8 \mathrm{~mm}$ and class $5 / 8 \mathrm{~mm}$, after a loading-unloading cycle, to the oedometric test.

After calculating $\mathrm{FD}_{\mathrm{R}}$ for the grains of different crushed samples, during the tests, under the different loads applied, the following results have been synthesized.

Due to the increase in the number of loading-unloading cycles (from 1 to 5 cycles), there was an offset of the curves in ascending order for all the samples studied in the oedometric tests. Actually, the higher the number of cycles, the greater the crushing of the grains, which induces an increase in the fractal dimension of roughness $\left(F D_{R}\right)$. The results of grains of limestone of sub-rounded shape are presented in the Figure 12.

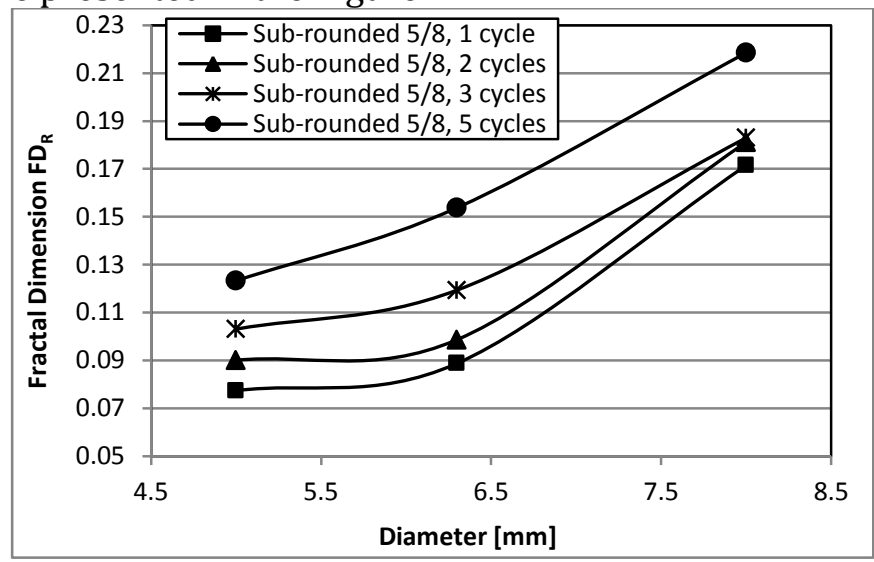

Fig 12. Variations of the fractal dimension roughness $\left(F D_{R}\right)$ of the class $5 / 8 \mathrm{~mm}$, for the sub-rounded limestone, depending on the number of loading cycles.

The Figure 13 shows that the granulometric class $5 / 8 \mathrm{~mm}$ crushes more than class $3.15 / 8 \mathrm{~mm}$, which crashes more than class $3.15 / 5 \mathrm{~mm}$. The calculated $\mathrm{FD}_{\mathrm{R}}$ increases with increasing grain size (grain diameter), regardless of the granular class and the number of cycles.

The production of fines during the tests is greater in the case of grains of large diameters ( 8 and $6.3 \mathrm{~mm}$ ) than in the case of small diameter grains (4 and $3.15 \mathrm{~mm}$ ). Grain $8 \mathrm{~mm}$ underwent a greater crushing, due to rupture of angularity by abrasion; On the other hand for the small grains of 3.15 and $4 \mathrm{~mm}$, it is a rupture by chipping which caused the increase in the roughness of the grains. This result is explained by the fact that the smaller grains are more resistant than the largest. Indeed, the larger the size, the greater the probability of areas of weakness (or cracking) in the grain.

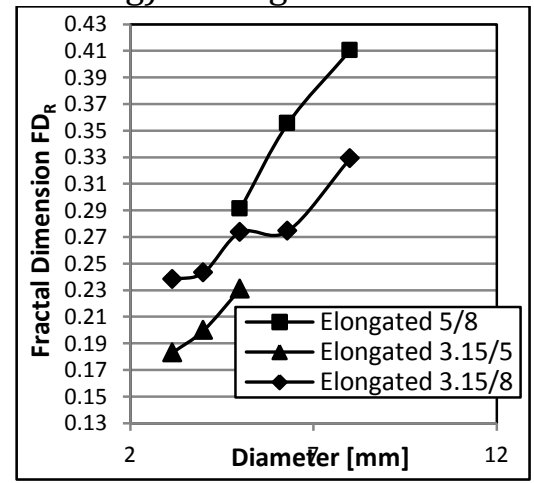

(a)

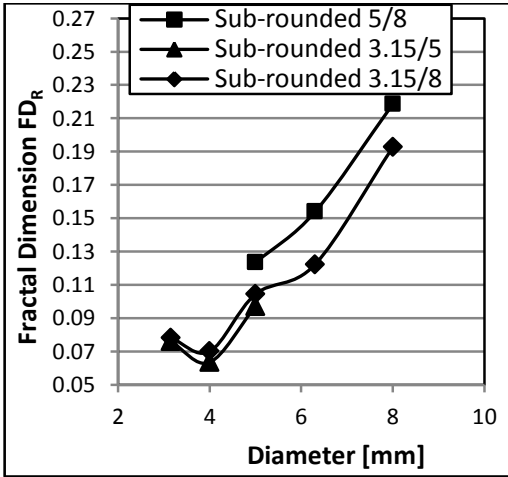

(b)

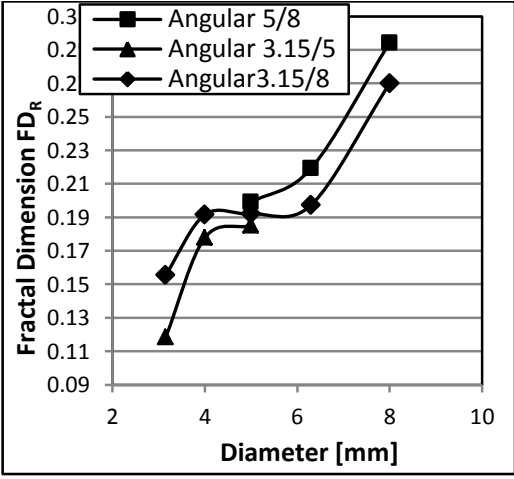

(c)

Fig 13. Variations of $F D_{R}$ as a function of the diameters analyzed, for the three granular classes: (a) elongated, (b) sub-rounded and (c) angular of the limestone, subjected to 5 cycles of loading-unloading to the oedometric test. 
The evolution of the fractal dimension of the elongated grains of limestone has higher values than those of sub-rounded and angular grain samples, regardless of the number of loadingunloading cycles and the applied stress. This can be explained by the crushing rate which is greater for the elongated form than for the other two forms studied (Figure 14).

The elongated shape has a rather large effect on the evolution of the fractal dimension for limestone (Figure 14).

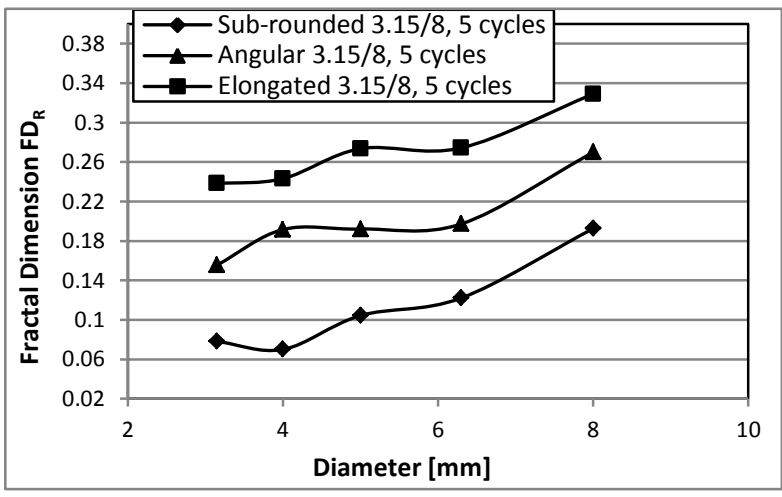

(a)

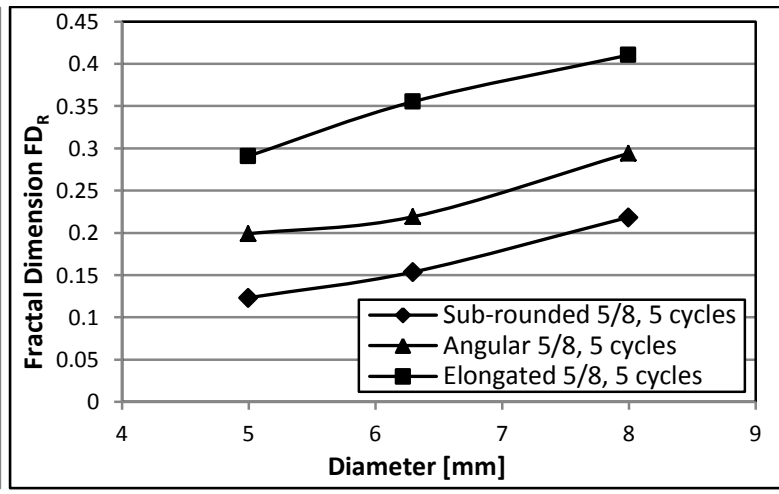

(b)

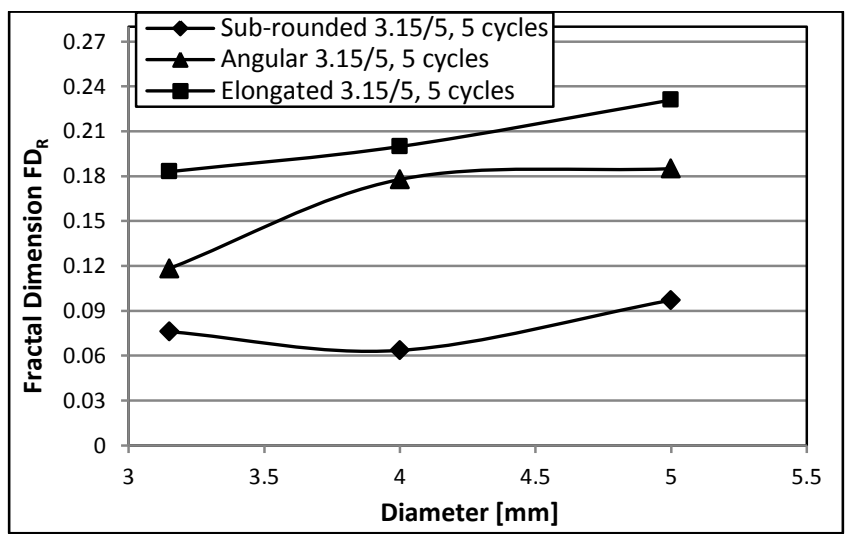

(c)

Fig 14. Variations of $F D_{R}$ as a function of the diameters analyzed, for the three forms limestone of classes : (a) $3.15 / 8$, (b) $5 / 8$ and (c) $3.15 / 5 \mathrm{~mm}$, subjected to five cycles of loading-unloading.

\section{Conclusions}

It has been determined that with an increase in the content of fines the fractal dimension increases. The oedometric test generates more grain rupture, which leads to a large variation in the fractal dimension. This allowed us to relate the fractal dimension to the number of loadingunloading cycles. These ratios were compared according to the amount of fines produced, the granulometric class, and the size and shape of the grains.

The variance of these last parameters (shapes, sizes and granular classes) significantly affects the rupture mode of the grains and their crushing; consequently, it increases or decreases the irregularities thereof. The three parameters mentioned above therefore affect their final fractal dimensions.

The analysis of the fractal dimension of grain images seems to be a powerful tool for comparing the intrinsic characteristics of the granular materials associated with their fragmentation. So, the algorithms provide a satisfactory estimate of the fractal dimension of the grains. 
These results make it possible, on the one hand, to characterize and understand the behavior of granular materials, on the other hand, to choose the right materials according to the function to be ensured in the works and the progress of civil engineering (dams, roads, watertight barriers, ballasted columns, concrete, etc).

It is recommended to improve this method of calculation and also to apply other methods of calculating the 3D fractal dimension of this material and other materials, subjected to various stresses to better understand the behavior of granular materials.

\section{References}

AFNOR (1997). Essai oedométrique. Essai de compressibilité sur les matériaux fins quasi saturés avec chargement par paliers. XPP 94-090-1, p 24.

Bouzeboudja, A., \& Melbouci, B. (2016a). Study of the evolution of the fractal dimension of a granular material's grains during mechanical tests. Bulletin of Engineering Geology and the Environment, Volume 75, Issue 2, pp 821-839.

Bouzeboudja, A. , Melbouci, B., \& Bouzeboudja, H. (2016b). Characterization of aggregates fragmentation using the calculation of fractal dimension" 16th International Conference on New Trends in Fatigue and Fracture (NT2F16), May 24-27, 2016, Dubrovnik, Croatia, ISBN 978-953-7738-39-6, pp. 211-213.

Chen, J., Lo, K.Y., Leung, H., \& Litva, J. (1996). The Use of Fractals for Modeling EM Waves Scattering from Rough Sea Surface. IEEE Transactions On Geoscience and Remote Sensing, Vol. 34, NO. 4.

Einav, I. (2007a). Breakage mechanics. Part I: Theory. J. Mech. Phys. Solids 55(6), 1274-1297.

Falconer, K., Chichester, Wiley, J., \& Sons. (1990). Fractal Geometry: Mathematical Foundations and Applications. 2e éd, poche (ISBN 978-0 470-84862-3) (LCCN 2004271361).

Huang, G.H., \& Zhan, W.H. (2002). Fractal property of soil particle size distribution and its application. Acte Pedologica Sinica, 39, pp. 490-497.

Lade, P.V., Yamamuro, J.A., et al. (1996). Significance of particule crushing in granular materials. Journal de la technologie géotechnique, vol.122, $\mathrm{N}^{\circ}$ 4, pp. 3109-3116.

Mandelbrot, B. (1983). The fractal geometry of nature. Edition W.II. Freeman, San Francisco, 461 pp.

Ovalle, C. (2013). contribution a l'étude de la rupture des grains dans les matériaux granulaires. MECHANICS OF MATERIALS. Ecole Centrale de Nantes (ECN), French.

Ramamurthy, T. (1969). Crushing Phenomena in Granular Soils. Journal of the Indian National Society of Soil Mechanics and Foundation Eng. Vol. 8.

Russel, D., Hanson, J., \& OTT, E. (1980). Dimension of strange attractors. Physical review Letters 45, 1175 1178.

Sezer, A., Altun, S., \& Erdogan, D. (2008). Correlation between CBR Strength and fractal Dimensions of Sands. 12eme Conference international de (IACMAG), 1-6 October, Goa Indian.

Wang, X., LI, M-H., Lui, S., \& Lui, G. (2006). Fractal characteristics of soils under different land-usepatterns in the arid and semiarid regions of the Tibetan plateau. China, Geoderma, 134 (1-2), pp. 56-61. 